Proceedings of the

International Geometry Center

Vol. 13, no. 4 (2020) pp. 40-49

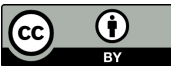

\title{
Olympic links in a Chebotarev link
}

\author{
Jun Ueki
}

\begin{abstract}
The Chebotarev law for an infinite link is an equidistribution property about how its components are linked in a group theoretic sense. We overview several properties of a Chebotarev link following the author's article "Chebotarev links are stably generic". In addition, we exhibit the density of modulo 2 Olympic links in a Chebotarev link.
\end{abstract}

Анотація. Закон Чеботарева для нескінченних завузлень це властивість рівномірності розподілу того, як кола завузлені з точки зору теорії груп. Ми розглядаємо кілька властивостей завузлення Чеботарева, слідуючи статті автора "Завузлення Чеботарева є стабільно типовими". Крім того, ми показуємо щільність по модулю 2 «олімпійського завузленнь» в завузленнях Чеботарева.

\section{INTRODUCTION}

The analogy between knots and prime numbers was initially pointed out by B. Mazur [13] and developed in a systematic manner by M. Kapranov [9], A. Reznikov [23,24], M. Morishita [18,19] and others. The Chebotarev law for an infinite link is an equidistribution property about how its components are linked in a group theoretic sense. An infinite link in $S^{3}$ obeying the Chebotarev law might be a good analogue of the set of all prime numbers in several senses. In this article, we overview several properties of Chebotarev links mainly following the author's article [32] and also exhibit the density of modulo 2 Olympic links in a Chebotarev link as a new example of Chebotarev phenomenon.

We first overview the relationship between two analogues in a 3-manifold of the set of rational prime numbers. We recall the definitions and state that if $\left(K_{i}\right)_{i \in \mathbb{N}_{>0}}$ is a sequence of knots obeying the Chebotarev law in the sense of B. Mazur and C. T. McMullen, then $\mathcal{K}=\cup_{i} K_{i}$ is a stably generic

Keywords: link, 3-manifold, pseudo-Anosov flow, idelic class field theory

Ключові слова: зачеплення, 3-многовид, псевдо-аносівсткий потік, теорія ідеальних класів полів

DOI: http://dx.doi.org/10.15673/tmgc.v13i4.1751 
link in the sense of T. Mihara. We outline the proof and also remark our perspective on Idelic class field theory on a 3-manifold and Artin $L$ functions.

Next, we recall McMullen's examples and discuss the planetary links obtained from fibered hyperbolic links in $S^{3}$. In addition, we recall an analogue of Artin's argument and exhibit the decomposition table in an analogue of a quintic field.

Finally, we recall the notions of (modulo 2) Olympic primes and links and calculate the density of modulo 2 Olympic links in a Chebotarev link.

\section{Chebotarev Links are Stably GeneriC}

Definitions and Theorems. We assume that any 3-manifold is the complement of a finite link in an oriented connected closed 3-manifold. A knot $K$ in a 3 -manifold $M$ means a tame embedding $S^{1}=\mathbb{R} / \mathbb{Z} \hookrightarrow M$ or its image with a natural orientation. A link is a countable (finite or infinite) set of disjoint knots. For any manifold $X$, we denote the interior of $X$ by $\operatorname{Int} X$.

McMullen [15] established a version of the Chebotarev density theorem in which number fields are replaced by 3-manifolds, answering to Mazur's question on the existence of Chebotarev arrangement of knots in [14]. Their definition is described as follows.

Definition 2.1 (Chebotarev law). Let $\left(K_{i}\right)=\left(K_{i}\right)_{i \in \mathbb{N}_{>0}}$ be a sequence of disjoint knots in a 3-manifold $M$. For each $n \in \mathbb{N}_{>0}$ and $j>n$, we put $L_{n}=\cup_{i \leqslant n} K_{n}$ and denote the conjugacy class of $K_{j}$ in $\pi_{1}\left(M-L_{n}\right)$ by $\left[K_{j}\right]$. We say that $\left(K_{i}\right)$ obeys the Chebotarev law if

$$
\lim _{\nu \rightarrow \infty} \frac{\#\left\{n<j \leqslant \nu \mid \rho\left(\left[K_{j}\right]\right)=C\right\}}{\nu}=\frac{\# C}{\# G}
$$

holds for any $n \in \mathbb{N}_{>0}$, any surjective homomorphism $\rho: \pi_{1}\left(M-L_{n}\right) \rightarrow G$ to any finite group, and any conjugacy class $C \subset G$. (The left hand side is the natural density of $K_{i}$ 's with $\rho\left(\left[K_{j}\right]\right)=C$.)

An infinite link $\mathcal{K}$ is said to be Chebotarev if it obeys the Chebotarev law with respect to some order.

On the other hand, Mihara [16] formulated an analogue of idelic class field theory for 3-manifolds by introducing certain infinite links called stably generic links, refining the notion of very admissible links given by Niibo and the author [20,21], and gave a cohomological interpretation of our previous formulation. Here we describe the definition of a stably generic link, only using ordinary terminology of low dimensional topology: 
Definition 2.2 (stably generic link). Let $M$ be a 3 -manifold and $\mathcal{K} \neq \varnothing$ a link. The link $\mathcal{K}$ is said to be generic if for any finite sublink $L$ of $\mathcal{K}$, the group $H_{1}(M-L)$ is generated by components of $\mathcal{K}-L$. The link $\mathcal{K}$ is said to be stably generic if for any finite sublink $L$ of $\mathcal{K}$ and for any finite branched cover $h: M^{\prime} \rightarrow M$ branched over $L$, the preimage $h^{-1}(\mathcal{K})$ is again a generic link of $M^{\prime}$.

The following theorem is due to the author [32, Theorem 3]:

Theorem 2.3. Let $\left(K_{i}\right)$ be a sequence of disjoint knots in a 3-manifold $M$ obeying the Chebotarev law. Then the link $\mathcal{K}=\cup_{i} K_{i}$ is a stably generic link.

Remark 2.4. For a sequence of knots ordered by length and obeying the Chebotarev law, we may define analogues of Artin $L$-functions (cf. $[1,22,27$, 28]). In addition, Mihara's refinement allows us to study analogues of ray class fields. We expect that Theorem 2.3 would play a key role to expand an analogue of idèlic class field theory for 3-manifolds, in a direction of analytic number theory, with ample interesting examples. Another analogue in a more general setting are due to J. Kim (see ver.1 of [10]) and others [11].

Outline of the proof. A careful observation of the behavior of knots in a finite cover which is not necessarily Galois (regular) and the lifting property of a continuous map yield the following lemma.

Lemma 2.5. Let $h: N \rightarrow M$ be a finite (unbranched) cover of 3-manifolds and $K \subset M$ a knot.

(1) Let $K^{\prime}$ be a connected component of $h^{-1}(K)$ in $N$. If the restriction map $\left.h\right|_{K^{\prime}}: K^{\prime} \rightarrow K$ is a bijection, then the conjugacy classes satisfy $h_{*}\left(\left[K^{\prime}\right]\right) \subset[K]$ in $\pi_{1}(M)$.

(2) If $k \in[K] \cap h_{*}\left(\pi_{1}(N)\right) \neq \varnothing$, then there exists some connected component $K^{\prime}$ of $h^{-1}(K)$ such that $\left.h\right|_{K^{\prime}}: K^{\prime} \rightarrow K$ is a bijection and $k \in h_{*}\left(\left[K^{\prime}\right]\right)$ holds.

We have an analogue of the Hilbert ramification theory for Galois branched covers of 3-manifolds, in which we describe the behavior of knots (instead of prime ideals) using the language of fundamental groups [31]. For a non-Galois cover $h: N \rightarrow M$ and a knot $K$ in $M$, the covering degrees of the restriction maps $\left.h\right|_{K_{i}^{\prime}}: K_{i}^{\prime} \rightarrow K$ for components of $h^{-1}(K)=\cup_{i} K_{i}^{\prime}$ does not necessarily coincide with each other.

In order to prove Theorem 2.3, we introduce the notion of a weakly Chebotarev link: 
Definition 2.6. Let $M$ be a 3 -manifold and $\mathcal{K}=\cup_{i \in \mathbb{N}_{>0}} K_{i}$ a countable link. The link $\mathcal{K}$ in $M$ is said to be weakly Chebotarev if for any surjective homomorphism $\rho: \pi_{1}(M) \rightarrow G$ to any finite group, any conjugacy class $C$ of $G$ is the image $\rho\left(\left[K_{i}\right]\right)$ of the conjugacy class $\left[K_{i}\right] \subset \pi_{1}(M)$ of some component $K_{i}$ of $\mathcal{K}$.

Lemma 2.5 yields the following key lemma.

Lemma 2.7. If $\mathcal{K}=\cup_{i} K_{i}$ in $M$ is weakly Chebotarev, then for any finite (unbranched) cover $h: N \rightarrow M$, the preimage $\mathcal{K}^{\prime}=\cup_{j} K_{j}^{\prime}$ of $\mathcal{K}$ is again weakly Chebotarev.

We also have the following lemma.

Lemma 2.8. If a link $\mathcal{K}=\cup_{i} K_{i}$ in a 3-manifold $M$ is weakly Chebotarev, then $H_{1}(M)$ is generated by components of $\mathcal{K}$.

Now Lemmas 2.7, 2.8, and a careful reading of the definition of a stably generic link yield Theorem 2.3.

\section{Examples of Chebotarev links}

McMullen's results. McMullen proved that sequences of knots

$$
\left(K_{i}\right)=\left(K_{i}\right)_{i \in \mathbb{N}_{>0}}
$$

given in Examples 3.1 below obey the Chebotarev law [15, Theorems 1.1, 1.2]. Hence the union $\mathcal{K}=\cup_{i} K_{i}$ of such $\left(K_{i}\right)$ is a stable generic link by Theorem 2.3.

Examples 3.1. (1) Let $X$ be a closed surface of constant negative curvature, let $M=T_{1}(X)$ denote the unit tangent bundle, and let $\left(K_{i}\right)$ denote the closed orbits of the geodesic flow in $M$, ordered by length.

(2) Let $\left(K_{i}\right)$ be the closed orbits of any topologically mixing pseudoAnosov flow on a closed 3-manifold $M$, ordered by length in a generic metric. We consult $[4,5]$ for terminology and basic facts related to pseudoAnosov flows.

In [15], in order to connect symbolic dynamics to finite branched covers, McMullen proved an important lemma that assures that every conjugacy class of $G$ is presented by a closed orbit, and invoked the notion of a Markov section. Then he applied a Chebotarev law for dynamical setting, which was proved by Parry-Pollicott [22, Theorem 8.5] with use of a method of Artin $L$-functions. We note that special cases of (1) and (2) were initially proved to obey the Chebotarev law by Adachi and Sunada in [27, Proposition II2-12] and [1, Proposition C], the latter being related to topological entropy. 
Planetary links. An interesting example contained in (2) above is the planetary link $\mathcal{K}$ of a fibered hyperbolic finite link $L$ in $S^{3}$ introduced by Birman and Williams [3], that is, the periodic orbits of the suspension flow of the monodromy map of $L$. By virtue of McMullen's theorem [15, Theorem 1.2] together with the Nilsen-Thurston uniformization theorem [30, Theorem 0.1], the union $\mathcal{K} \cup L$ obeys the Chebotarev law, if ordered by length. We may prove in a similar way to Miller [17, Proposition 4.2] that for any finite sublink $L^{\prime} \subset \mathcal{K}$, the union $L \cup L^{\prime}$ is again hyperbolic. Moreover, we may prove that such $\mathcal{K} \cup L$ is stably Chebotarev. See [32, Section 4].

In addition to the Chebotarev law, the planetary link sometimes has another very noteworthy property. Ghrist and others proved that if a link $L$ belongs to a certain large class of links containing the figure-eight knot, then the planetary link of $L$ contains every links [6-8]. Hence we have a sequence $\left(K_{i}\right)$ of knots containing every isotopy class of links and obeying the Chebotarev law. Moreover, as formulated by Kopei in [12], this example satisfies an analogue of the product formula $|a|_{\infty} \prod_{p}|a|_{p}=1(a \in \overline{\mathbb{Q}})$, where $p$ runs through all the prime numbers and $|a|_{p}$ denote the $p$-adic norm with $|p|_{p}=p^{-1}$. Therefore, the planetary link would give a fundamental setting, when we establish an analogue of number theory on 3-manifolds.

\section{Artin's Argument for $A_{5}$-EXtensions}

Here we discuss an analogue of a quintic field. We first state the coincidence of the decomposition type of a knot and the cycle type of the monodromy permutation, which is an analogue of Artin's argument in [2] (see also [29, Chapter 16.2, Theorem 2]).

Definition 4.1. (1) For each $n \in \mathbb{N}_{>0}$, we denote the $n$-th symmetric group by $S_{n}$ and the $n$-th alternating group by $A_{n}$. The cycle type of $\sigma \in S_{n}$ is $\left(f_{1}, \cdots, f_{r}\right)$ if it is the product of disjoint cycles of length $f_{1} \geqslant \cdots \geqslant f_{r}$.

(2) For a finite cover $h: N \rightarrow M$ and a knot $K \subset M$, the decomposition type of $K$ in $h$ is $\left(f_{1}, \cdots, f_{r}\right)$ if the inverse image of $K$ consists of $r$ components as

$$
h^{-1}(K)=\underset{1 \leqslant i \leqslant r}{\cup} K_{i}
$$

with $f_{i}=\operatorname{deg}\left(h: K_{i} \rightarrow K\right)$ for $i=1, \cdots, r$ and $f_{1} \geqslant \cdots \geqslant f_{r}$.

We may prove the following proposition in a parallel way to the one in number theory.

Proposition 4.2. Let $\tilde{h}: W \rightarrow M$ be a finite (unbranched) Galois cover with $G=\operatorname{Gal}(\tilde{h})$, and $h: N \rightarrow M$ a subcover of degree $n$, which is not necessarily Galois. Let $\rho: G \rightarrow S_{n}$ denote the monodromy permutation 
induced by putting

$$
h^{-1}\left(b_{M}\right)=\left\{b_{1}, b_{2}, \cdots, b_{n}\right\}
$$

with $b_{1}=b_{N}$.

Let $K \subset M$ be a knot and let $z \in G$ be an element of the image of the conjugacy class $[K] \subset \pi_{1}(M)$ of $K$ under the natural homomorphism

$$
\pi_{1}(M) \rightarrow \pi_{1}(M) / \tilde{h}_{*}\left(\pi_{1}(W)\right) \cong G .
$$

Then the cycle type of $\rho(z)$ is $\left(f_{1}, \cdots, f_{r}\right)$ if and only if the decomposition type of $K$ in $h$ is $\left(f_{1}, \cdots, f_{r}\right)$.

Now we observe the density of knots in a Chebotarev link of each decomposition type in a degree 5 subcover of an $A_{5}$ (icosahedral)-cover.

Example 4.3. Let $\mathcal{K}=\cup_{i} K_{i}$ be the planetary link of the figure-eight knot in $S^{3}$, obeying the Chebotarev law. Let $L$ be a trefoil in $\mathcal{K}$, and put $M=S^{3}-L$. We have a well-known surjective homomorphism

$$
\tau: \pi_{1}(M) \rightarrow A_{5} \text {. }
$$

Let $\tilde{h}: W \rightarrow M$ denote the corresponding $A_{5}$-cover. (Then the Fox completion $\bar{W}$ of $W$ is a Poincaré 3-sphere, cf. [25].) Let $H<\operatorname{Gal}(h) \cong A_{5}$ be any subgroup of index 5, and let $h: N \rightarrow M$ denote the corresponding subcover of degree 5 . The kernel $\operatorname{Ker}(\rho)$ of the monodromy permutation $\rho: A_{5} \rightarrow S_{5}$ coincides with the normalizer of $H$ in $A_{5}$. Since $A_{5}$ is a simple group, $\operatorname{Ker}(\rho)=\{\mathrm{id}\}$ holds and $\rho$ is an injection. By Proposition 4.2 together with the Chebotarev law applied for the composite

$$
\rho^{\prime}: \pi_{1}(M) \underset{\tau}{\rightarrow} A_{5} \underset{\rho}{\stackrel{\cong}{\leftrightarrows}} \operatorname{Im}(\rho),
$$

the number of elements of $A_{5}$ of each cycle type and the natural density of $K_{i}$ 's of each decomposition type are given as follows.

\begin{tabular}{|c||c|c|c|c|}
\hline (cycle/decomposition) type & $(1,1,1,1,1)$ & $(2,2,1)$ & $(3,1,1)$ & $(5)$ \\
\hline \hline number of elements of $A_{5}$ & 1 & 15 & 20 & 24 \\
\hline density of knots in $\mathcal{K}$ & $1 / 60$ & $1 / 4$ & $1 / 3$ & $2 / 5$ \\
\hline
\end{tabular}

Here, a knot of decomposition type $(1,1,1,1,1)$ is totally decomposed and that of (5) is totally inert. The natural density of $K_{i}$ 's with property $P$ is defined by

$$
\lim _{\nu \rightarrow \infty} \frac{\#\left\{i \leqslant \nu \mid K_{i} \text { satisfies } P\right\}}{\nu} .
$$

Artin $L$-functions of symbolic flows due to Parry-Pollicott [22] are regarded as that of Chebotarev links. We may also discuss an analogue of Artin's argument in [2] with use of $L$-functions associated to the setting in Example 4.3. 


\section{OLYMPIC PRIMES AND LINKS}

The analogy between the Legendre symbol $\left(\frac{p}{q}\right)$ for two prime numbers $p, q$ and the modulo 2 linking number $\operatorname{lk}_{2}(K, L)$ of a two-component link $K \sqcup L$ is the most classical one in the dictionary of arithmetic topology. Some people say that it was initially noticed by Gauss, and indeed we may find some ideas in his proofs of the quadratic reciprocity $\left(\frac{q^{*}}{p}\right)=\left(\frac{p}{q}\right)$ for $q^{*}=(-1)^{\frac{q-1}{2}} q$ which might have come from his study in electromagnetism.

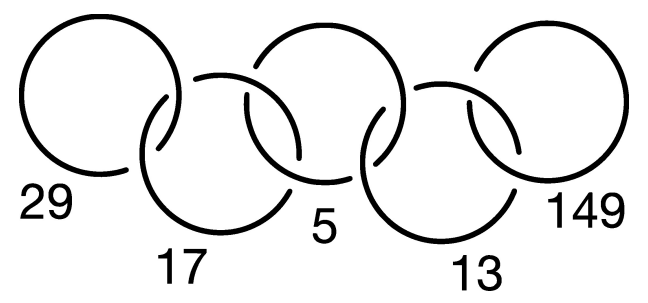

An Olympic prime is a 5 -tuple $\left(p_{1}, p_{2}, p_{3}, p_{4}, p_{5}\right)$ of prime numbers satisfying

$$
\left(\frac{p_{i+1}^{*}}{p_{i}}\right)=-1 \text { for } i=1,2,3,4 \quad \text { and } \quad\left(\frac{p_{j}^{*}}{p_{i}}\right)=1 \text { for } i+1<j .
$$

An example is displayed in the figure above. On the first day of Morishita's course in arithmetic topology for undergrads in October 2012, he asked to prove that there are infinitely many Olympic primes. By using Dirichlet's Theorem [26, Chapter VI, Theorem 2], for instance, we may easily calculate that the natural density of Olympic primes is $d=\frac{1}{2^{10}}$, where $10=\left(\begin{array}{l}5 \\ 2\end{array}\right)$ is "5 choose 2".

Similarly, a modulo 2 Olympic link is a link $L_{1} \cup \cdots \cup L_{5}$ with 5 components in $S^{3}$ satisfying

$$
\operatorname{lk}_{2}\left(L_{i}, L_{i+1}\right)=1 \text { for } i=1,2,3,4 \text { and } \quad \mathrm{lk}_{2}\left(L_{i}, L_{j}\right)=0 \text { for } i+1<j .
$$

Now let $\left(K_{i}\right)_{i \in \mathbb{N}>0}$ be a sequence of disjoint knots in $S^{3}$ obeying the Chebotarev law and put $\mathcal{K}=\underset{i \in \mathbb{N}_{>0}}{\cup} K_{i}$. For each $n \in \mathbb{N}_{>0}$, let $\underset{1 \leqslant i \leqslant n}{\cup} L_{i}$ be an $n$-component sublink of $\mathcal{K}$ and consider the surjective homomorphism

$$
\begin{gathered}
\rho: \pi_{1}\left(S^{3}-\cup_{1 \leqslant i \leqslant n} L_{i}\right) \rightarrow(\mathbb{Z} / 2 \mathbb{Z})^{n} \\
{\left[K_{j}\right] \longmapsto\left(\operatorname{lk}_{2}\left(L_{i}, K_{j}\right)\right)_{i \in\{1,2, \ldots, n\}} .}
\end{gathered}
$$

Then the Chebotarev law yields that the natural density of $K_{j}$ 's with $\rho\left(\left[K_{j}\right]\right)=(0, \cdots, 0,1)$ is $d_{n}=\frac{1}{2^{n}}$. This fact for $n=1,2,3,4$ yields that 
the natural density of modulo 2 Olympic links in $\mathcal{K}$ is

$$
d=\prod_{n \leqslant 4} d_{n}=\frac{1}{2^{10}}
$$

The similar argument holds for modulo $m$ linking number for every positive integer $m$. However, this does not tell whether there exists an Olympic link without modulo.

It might be also interesting to investigate the density of 3-component links with given Milnor's triple linking number.

\section{ACKNOWLEDGMENTS}

I would like to express my sincere gratitude to Sergey Maksymenko for inviting me to the workshop "Morse theory and its applications" in Kyiv, Ukraine. I was much inspired and encouraged by the atmosphere and culture there. I am also grateful to people who I met in Göttingen, Germany. When I visited the Gaußhaus, I felt that it is natural to expect his intuition of electromagnetism among prime numbers. Finally I would like to thank Hirofumi Niibo for asking an important question to clarify the argument in this manuscript, and the guy who gave me a copy of the lecture note containing the handwriting version of the figure above.

\section{REFERENCES}

[1] Toshiaki Adachi, Toshikazu Sunada. Twisted Perron-Frobenius theorem and Lfunctions. J. Funct. Anal., 71(1):1-46, 1987, doi: 10.1016/0022-1236(87)90014-0.

[2] E. Artin. Über die Zetafunktionen gewisser algebraischer Zahlkörper. Math. Ann., 89(12):147-156, 1923, doi: 10.1007/BF01448095.

[3] Joan S. Birman, R. F. Williams. Knotted periodic orbits in dynamical system. II. Knot holders for fibered knots. In Low-dimensional topology (San Francisco, Calif., 1981), volume 20 of Contemp. Math., pages 1-60. Amer. Math. Soc., Providence, RI, 1983, doi: $10.1090 /$ conm $/ 020 / 718132$.

[4] Danny Calegari. Foliations and the geometry of 3-manifolds. Oxford Mathematical Monographs. Oxford University Press, Oxford, 2007.

[5] Sérgio R. Fenley. Asymptotic geometry of foliations and pseudo-Anosov flows - a survey. In Groups of diffeomorphisms, volume 52 of Adv. Stud. Pure Math., pages 1-20. Math. Soc. Japan, Tokyo, 2008.

[6] Robert Ghrist, Eiko Kin. Flowlines transverse to knot and link fibrations. Pacific J. Math., 217(1):61-86, 2004, doi: 10.2140/pjm.2004.217.61.

[7] Robert W. Ghrist. Branched two-manifolds supporting all links. Topology, 36(2):423448, 1997, doi: 10.1016/0040-9383(96)00006-7.

[8] Robert W. Ghrist, Philip J. Holmes, Michael C. Sullivan. Knots and links in threedimensional flows, volume 1654 of Lecture Notes in Mathematics. Springer-Verlag, Berlin, 1997, doi: 10.1007/BFb0093387.

[9] M. M. Kapranov. Analogies between the Langlands correspondence and topological quantum field theory. In Functional analysis on the eve of the 21st century, Vol. 1 
(New Brunswick, NJ, 1993), volume 131 of Progr. Math., pages 119-151. Birkhäuser Boston, Boston, MA, 1995.

[10] Junhyeong Kim. On the leafwise cohomology and dynamical zeta functions for fiber bundles over the circle. arXiv:1712.04181v2, May 2018.

[11] Junhyeong Kim, Masanori Morishita, Takeo Noda, Yuji Terashima. On 3-dimensional foliated dynamical systems and hilbert type reciprocity law. arXiv:1906.02424, 2019.

[12] Fabian Kopei. A remark on a relation between foliations and number theory. In Foliations 2005, pages 245-249. World Sci. Publ., Hackensack, NJ, 2006, doi: 10.1142/9789812772640_0013.

[13] Barry Mazur. Remarks on the Alexander polynomial. http://www.math.harvard.edu/ 〜mazur/papers/alexander_polynomial.pdf, 1963-64.

[14] Barry Mazur. Primes, Knots and Po. Lecture notes for the conference "Geometry, Topology and Group Theory" in honor of the 80th birthday of Valentin Poenaru, July 2012.

[15] Curtis T. McMullen. Knots which behave like the prime numbers. Compos. Math., 149(8):1235-1244, 2013, https://doi.org/10.1112/S0010437X13007173.

[16] Tomoki Mihara. Cohomological approach to class field theory in arithmetic topology. Canad. J. Math., 71(4):891-935, 2019, doi: 10.4153/cjm-2018-020-0.

[17] Sally M. Miller. Geodesic knots in the figure-eight knot complement. Experiment. Math., 10(3):419-436, 2001, http://projecteuclid.org/euclid.em/1069786348.

[18] Masanori Morishita. On certain analogies between knots and primes. J. Reine Angew. Math., 550:141-167, 2002, doi: 10.1515/crll.2002.070.

[19] Masanori Morishita. Knots and primes. Universitext. Springer, London, 2012, doi: 10.1007/978-1-4471-2158-9. An introduction to arithmetic topology.

[20] Hirofumi Niibo. Idèlic class field theory for 3-manifolds. Kyushu J. Math, 68(2):421436, 2014.

[21] Hirofumi Niibo, Jun Ueki. Idèlic class field theory for 3-manifolds and very admissible links. Trans. Amer. Math. Soc., 371(12):8467-8488, 2019, doi: 10.1090/tran/7480.

[22] William Parry, Mark Pollicott. Zeta functions and the periodic orbit structure of hyperbolic dynamics. Astérisque, (187-188):268, 1990.

[23] Alexander Reznikov. Three-manifolds class field theory (homology of coverings for a nonvirtually $b_{1}$-positive manifold). Selecta Math. (N.S.), 3(3):361-399, 1997, doi: $10.1007 / \mathrm{s} 000290050015$.

[24] Alexander Reznikov. Embedded incompressible surfaces and homology of ramified coverings of three-manifolds. Selecta Math. (N.S.), 6(1):1-39, 2000, doi: $10.1007 / \mathrm{s} 000290050001$.

[25] Dale Rolfsen. Knots and links, volume 7 of Mathematics Lecture Series. Publish or Perish, Inc., Houston, TX, 1990. Corrected reprint of the 1976 original.

[26] J.-P. Serre. A course in arithmetic. Springer-Verlag, New York-Heidelberg, 1973. Translated from the French, Graduate Texts in Mathematics, No. 7.

[27] Toshikazu Sunada. Geodesic flows and geodesic random walks. In Geometry of geodesics and related topics (Tokyo, 1982), volume 3 of Adv. Stud. Pure Math., pages 47-85. North-Holland, Amsterdam, 1984.

[28] Toshikazu Sunada. Fundamental Groups and Laplacians. Kinokuniya, Tokyo, (in Japanese), 1988.

[29] Teiji Takagi. Daisūteki Seisūron. Gaisetsu oyobi Ruitairon. Iwanami Shoten, Tokyo, 1948.

[30] William P. Thurston. Hyperbolic structures on 3-manifolds, II: Surface groups and 3-manifolds which fiber over the circle. arXiv:math/9801019, 11986. 
[31] Jun Ueki. On the homology of branched coverings of 3-manifolds. Nagoya Math. J., 213:21-39, 2014.

[32] Jun Ueki. Chebotarev link are stably generic. to appear in Bull. Lond. Math. Soc., 2020, doi: 10.1112/blms.12400. arXiv:1902.06906.

Received: February 1, 2020, accepted: April 21, 2020.

Jun Ueki

Department of Mathematics, School of System Design and Technology, Tokyo

Denki University 5 Senju Asahi-Cho, Adachi-Ku, 120-8551, Tokyo, Japan

Email: uekijun46@gmail.com 